\title{
A Small-Sample Adaptive Hybrid Model for Annual Electricity Consumption Forecasting
}

\author{
Ming Meng, ${ }^{1}$ Yanan Fu, ${ }^{1}$ Huifeng Shi, ${ }^{2}$ and Xinfang Wang ${ }^{3}$ \\ ${ }^{1}$ Department of Economics and Management, North China Electric Power University, Baoding, Hebei 071003, China \\ ${ }^{2}$ Department of Mathematics and Physics, North China Electric Power University, Baoding, Hebei 071003, China \\ ${ }^{3}$ Tyndall Centre for Climate Change Research, University of Manchester, Manchester M13 9PL, UK \\ Correspondence should be addressed to Ming Meng; 51851341@ncepu.edu.cn
}

Received 20 February 2017; Revised 23 March 2017; Accepted 30 March 2017; Published 12 April 2017

Academic Editor: David González

Copyright (C) 2017 Ming Meng et al. This is an open access article distributed under the Creative Commons Attribution License, which permits unrestricted use, distribution, and reproduction in any medium, provided the original work is properly cited.

\begin{abstract}
Annual electricity consumption forecasting is one of the important foundations of power system planning. Considering that the long-term electricity consumption curves of developing countries usually present approximately exponential growth trends and linear and accelerated growth rate trends may also appear in certain periods, this paper first proposes a small-sample adaptive hybrid model (AHM) to extrapolate the above curves. The iterative trend extrapolation equation of the proposed model can simulate the linear, exponential, and steep trends adaptively at the same time. To estimate the equation parameters using small samples, the partial least squares (PLS) and iteration starting point optimization algorithms are suggested. To evaluate forecasting performance, the artificial neural network (ANN), grey model (GM), and AHM are used to forecast electricity consumption in China from 1991 to 2014, and then the results of these models are compared. Analysis of the forecasting results shows that the AHM can overcome stochastic changes and respond quickly to changes in the main electricity consumption trend because of its specialized equation structure. Overall error analysis indicators also show that AHM often obtains more precise forecasting results than the other two models.
\end{abstract}

\section{Introduction}

Given that electricity cannot be stored at a large scale, forecasting electricity consumption is important for power enterprises to balance supply and demand. Considering the differences among forecasting indicators, researches on electricity consumption forecasting are usually divided into two categories, namely, short-term and mid/long-term. Annual electricity consumption forecasting, which is usually used for power system planning, is categorized as mid/long-term.

For most developed countries that have achieved economic development at the postindustrialization stage and have fulfilled most of the electricity needs of people, their electricity consumption changes slowly and can be easily forecasted. By contrast, with the rapid development of the social economy, electricity consumption of developing countries usually changes rapidly and is difficult to forecast [1]. Since 2001 , the average yearly growth rate of the top 15 developed electricity consumers of the world is only $1.1 \%$, whereas that of 15 highest electricity consumers in developing nations or countries is as high as 7.4\% [2]. This study mainly focuses on the annual electricity consumption forecasting for developing countries.

At present, two ideas for annual electricity consumption forecasting have been proposed. Some researchers had focused on the relationship between annual electricity consumption and its influencing factors and designed multivariate equations to simulate this relationship, obtaining the forecasting results by inputting the values of the explanatory variable in the forecasting period into the equation [3-5]. However, as the exact values of the explanatory variable in the forecasting period are unknown, most models are usually only used for scenario forecasting. Many other researchers concentrated only on the change regularity of the annual electricity consumption trend. Equations are often used to simulate the annual electricity consumption curve and obtain 
the forecasting results by trend extrapolation [6-8]. These methods can obtain the "natural" change results of future electricity consumption. The adaptive hybrid model (AHM) proposed in this paper is a trend extrapolation method.

The long-term annual electricity consumption curves for most developing countries usually present increasing trends with different curvatures [2]. That is, these annual electricity consumption curves are often approximately exponential growth curves. The artificial neural network (ANN) and other similar models have been extensively used in trend extrapolation of short-term or monthly electricity consumption time series [9-11]. However, compared with these time series, annual electricity consumption has fewer data and, more importantly, is easily affected by socioeconomic environment changes. As such, many minor unexpected waves usually exist in its curve. In this manner, if ANN is used to extrapolate the annual electricity consumption, then the generalization ability of the trained network will easily be affected significantly. Furthermore, time series algorithms [12, 13] cannot easily handle situations wherein the sample size is small. The grey models (GM), which are represented by $\operatorname{GM}(1,1)$, have been successfully used in the trend extrapolation of the annual time series [14-16]. The merits of $\operatorname{GM}(1,1)$ are in the following two aspects. First advantage is that $\operatorname{GM}(1,1)$ can use small samples to estimate the equation parameters. Second, by adjusting the parameters, $\operatorname{GM}(1,1)$ can simulate the approximately exponential growth curve using any slope and curvature. However, when used to forecast annual electricity consumption, $\operatorname{GM}(1,1)$ still has the following limitations.

The forecasting equation of $\operatorname{GM}(1,1)$ is essentially a homogeneous exponential equation, which can only perfectly simulate the time series with a steady growth rate. This makes it unable to perfectly adapt the characteristics of the real annual electricity consumption data. For one thing, the feature of steady growth rate does not necessarily exist in annual electricity consumption data. For another, with the changes of the socioeconomic environment, the annual electricity consumption trend usually cannot remain steady for a long time. Except for the approximately exponential growth trend, the linear trend also usually appears in specific periods. Evidently, $\operatorname{GM}(1,1)$ cannot simulate this linear trend. Furthermore, as mentioned previously, the forecasting equation of $\operatorname{GM}(1,1)$ is essentially a homogeneous exponential equation. The structure of this equation has the upper limit of growth speed. As the social economy develops, the electricity consumption share in end-use energies generally increases gradually. As a result, the annual electricity consumption trend is usually steeper than the total energy consumption and $\mathrm{CO}_{2}$ emissions trends [17]. In the period of economic boom, the electricity consumption trend will be very steep and the growth rate of it may increase gradually. Evidently, $\operatorname{GM}(1,1)$ cannot handle this situation.

In this paper, the AHM is first proposed to forecast annual electricity consumption by trend extrapolation. The forecasting equation of this model contains linear, nonhomogeneous exponential, and steep items. By adjusting the equation parameters using small samples, this equation can simulate the above three trends adaptively at the same time.

\section{Hybrid Equation}

This section discusses the hybrid equation which can simulate linear, nonhomogeneous exponential, and steep trends at the same time.

The fitting and forecasting equation of $\operatorname{GM}(1,1)$ to $x_{k}[14-$ $16]$ is then written as

$$
\widehat{x}_{k}=\left(1-e^{\beta_{1}}\right)\left(x_{1}-\frac{\beta_{2}}{\beta_{1}}\right) e^{-\beta_{1}(k-1)},
$$

where $\beta_{1}$ and $\beta_{2}$ are the equation parameters, respectively.

Notably, (1) is a homogeneous exponential equation. Based on its analytical expression, the following relationship between $\widehat{x}_{k}$ and $\widehat{x}_{k-1}$ should exist:

$$
\frac{\widehat{x}_{k}}{\widehat{x}_{k-1}}=e^{-\beta_{1}} \text {. }
$$

As a result, (1) can be written as

$$
\begin{aligned}
& \widehat{x}_{k}=e^{-\beta_{1}} \widehat{x}_{k-1}, \\
& \widehat{x}_{1}=\left(1-e^{\beta_{1}}\right)\left(x_{1}-\frac{\beta_{2}}{\beta_{1}}\right) .
\end{aligned}
$$

Furthermore, as previously discussed, the steep trend that has an increasing growth rate may also appear in the annual electricity consumption time series. Considering that a linear growth rate can ensure a highly steep trend, the simulation equation of the steep trend can be written as follows:

$$
\frac{\widehat{x}_{k}}{\widehat{x}_{k-1}}=\gamma_{1}+\gamma_{2} k,
$$

where $\gamma_{1}$ and $\gamma_{2}$ are the equation parameters.

That is,

$$
\widehat{x}_{k}=\gamma_{1} \widehat{x}_{k-1}+\gamma_{2} k \widehat{x}_{k-1} \text {. }
$$

Based on (3) and (5) and with the assumption that the linear trend may also appear in the annual electricity consumption time series, the hybrid equation can be written as

$$
\begin{aligned}
\widehat{x}_{k+1} & =\lambda_{1}+\lambda_{2} k+\lambda_{3} \widehat{x}_{k}+\lambda_{4} k \widehat{x}_{k}, \\
\widehat{x}_{1} & =x_{1}+\lambda_{5} .
\end{aligned}
$$

The first part of (6) shows the iterative relationship between two adjacent values, while the second part ascertains the starting point of iteration. The iterative equation obviously contains linear, exponential, and steep items. This equation structure has the ability to simulate the three trends at the same time. Therefore, (6) is more applicable than (1) in forecasting the annual electricity consumption time series.

\section{Parameter Estimation}

3.1. Parameter Estimation of the Iterative Equation. Influenced by the changes in the socioeconomic environment, the annual electricity consumption time series cannot easily 
maintain a steady trend for a long time. That is, collecting a large number of samples to estimate the parameters of the iterative equation is nearly impossible. Moreover, considering the unique structure of the iterative equation, multicollinearity between the independent variables is serious, especially when the sample size is small. Multicollinearity will render the estimations obtained by the traditional ordinary least squares (OLS) as highly unstable. As such, the forecasting results will be with significant risks. Similar to OLS, the partial least squares (PLS) can also estimate the parameters of a linear equation. However, unlike OLS, PLS has the ability to determine the regression parameters using few observations with multicollinearity [18]. Hence, this study adopts the idea of PLS to design the parameter estimation algorithm of the previously presented iterative equation.

The key idea of PLS is to extract components to overcome the influence of multicollinearity. Before component extraction, preprocessing of the observations is essential.

Given the structure of (6), let

$$
\begin{aligned}
& F_{0}=\left(\frac{x_{k}-\operatorname{mean}\left(x_{k}\right)}{\operatorname{std}\left(x_{k}\right)}\right)_{n-1,1}, \quad k=2,3, \ldots, n, \\
& E_{0} \\
& =\left(\frac{k-\operatorname{mean}(k)}{\operatorname{std}(k)} \frac{x_{k}-\operatorname{mean}\left(x_{k}\right)}{\operatorname{std}\left(x_{k}\right)} \frac{k x_{k}-\operatorname{mean}\left(k x_{k}\right)}{\operatorname{std}\left(k x_{k}\right)}\right)_{n-1,3}, \\
& k=1,2, \ldots, n-1 ;
\end{aligned}
$$

the extracted component is obtained using the following equation $[19,20]$ :

$$
t_{1}=E_{0} w_{1}
$$

where $w_{1}=E_{0}^{T} F_{0} /\left\|E_{0}^{T} F_{0}\right\|$ and $\left\|w_{1}\right\|=1$.

Using $t_{1}$ as the independent variable to explain $F_{0}$ and $E_{0}$, the residuals are written as $F_{1}$ and $E_{1}$, respectively. That is,

$$
\begin{aligned}
& E_{0}=t_{1} p_{1}^{T}+E_{1}, \\
& F_{0}=t_{1} r_{1}+F_{1},
\end{aligned}
$$

where $p_{1}=E_{0}^{T} t_{1} /\left\|t_{1}\right\|^{2}$ and $r_{1}=F_{0}^{T} t_{1} /\left\|t_{1}\right\|^{2}$.

Replacing $F_{0}$ and $E_{0}$ in (8) with $F_{1}$ and $E_{1}$, respectively, the second component $t_{2}$ is obtained. Using a similar method, $t_{3}$ and $t_{4}$ are also obtained.

More extracted components do not necessarily result in better forecasting precision. In fact, if the current components already contain useful information, more components will introduce stochastic information into the final equation and accordingly reduce the generalization capability of the equation, although simulation precision may be improved. From the second component $t_{2}$, the importance of each component should be tested first before being introduced into the final equation. The indicator to evaluate the importance of a component is defined as follows:

$$
Q_{h}^{2}=1-\frac{\sum_{k=2}^{n}\left(\hat{x}_{h,-k}-x_{k}\right)^{2}}{\sum_{k=2}^{n}\left(\hat{x}_{h-1, k}-x_{k}\right)^{2}},
$$

where $\widehat{x}_{h,-k}$ is the forecasting result of $x_{k}$ by the forecasting equation, which is established using the first $h$ components and $n-1$ samples (the $k$ th sample is excluded), and $\widehat{x}_{h-1, k}$ is the forecasting result of $x_{k}$ by the forecasting equation, which is established using the first $h-1$ components and all samples.

Based on statistical experience, when $Q_{h}^{2} \geq 0.0975$, the $h$ th component should be introduced into the final equation [21]. Given that the importance of the extracted components decreases one after the other, if the $h$ th component is identified as unnecessary to be added into the equation, then the subsequent components $\left(t_{h+1}\right.$ and $t_{h+2}$ ) will unnecessarily be tested and should be abandoned directly.

Given that $l$ components remained, the explanatory equation to $F_{0}$ is written as

$$
\widehat{F}_{0}=r_{1} t_{1}+r_{2} t_{2}+\cdots+r_{l} t_{l} .
$$

As the extracted components have only slight multicollinearity, the regression parameters of (11) can be obtained by OLS. Using the inverse operation of preprocessing and component extraction, (11) can be transformed into the iterative equation of (6).

3.2. Parameter Estimation of the Starting Point. Equation (6) shows that the starting point of the iterative equation will affect the long-term simulation and forecasting results. To obtain the optimum estimation of $\lambda_{5}$ which can decide the starting point, the transformation to (6) is necessary.

Let

$$
\begin{aligned}
& a_{k}=\lambda_{1}+\lambda_{2} k, \\
& b_{k}=\lambda_{3}+\lambda_{4} k ;
\end{aligned}
$$

(6) is further written as

$$
\begin{aligned}
& \hat{x}_{1}=x_{1}+\lambda_{5}, \\
& \hat{x}_{2}=a_{1}+b_{1}\left(x_{1}+\lambda_{5}\right), \\
& \hat{x}_{3}=a_{2}+a_{1} b_{2}+b_{1} b_{2}\left(x_{1}+\lambda_{5}\right), \\
& \hat{x}_{4}=a_{3}+a_{2} b_{3}+a_{1} b_{2} b_{3}+b_{1} b_{2} b_{3}\left(x_{1}+\lambda_{5}\right)
\end{aligned}
$$

$$
\begin{aligned}
\widehat{x}_{k} & =a_{k-1}+a_{k-2} b_{k-1}+a_{k-3} b_{k-1} b_{k-2}+\cdots \\
& +a_{1} b_{k-1} b_{k-2} \cdots b_{2}+b_{1} b_{2} \cdots b_{k-1}\left(x_{1}+\lambda_{5}\right) .
\end{aligned}
$$

Let

$$
\begin{aligned}
c_{k}= & a_{k-1}+a_{k-2} b_{k-1}+a_{k-3} b_{k-1} b_{k-2}+\cdots \\
& +a_{1} b_{k-1} b_{k-2} \cdots b_{2}, \\
d_{k}= & \prod_{j=1}^{k-1} b_{j} ;
\end{aligned}
$$

(13) can be written as

$$
\begin{aligned}
& \widehat{x}_{1}=x_{1}+\lambda_{5}, \quad k=1, \\
& \widehat{x}_{k}=c_{k}+d_{k}\left(x_{1}+\lambda_{5}\right), \quad k>1 .
\end{aligned}
$$


Based on the general optimization idea, the optimum estimation of $\lambda_{5}$ should ascertain the residual sum of squares (RSS) to reach its minimum value.

Let

$$
F\left(\lambda_{5}\right)=\sum_{k=1}^{n}\left(\widehat{x}_{k}-x_{k}\right)^{2}
$$

be the RSS. The first derivative of $F\left(\lambda_{5}\right)$ with respect to $\lambda_{5}$ should be equal to 0 to obtain the minimum value. That is,

$$
\begin{aligned}
\frac{\partial F\left(\lambda_{5}\right)}{\partial \lambda_{5}} & =2 \lambda_{5}+2 \sum_{k=2}^{n}\left(\widehat{x}_{k}-x_{k}\right) \frac{\partial \widehat{x}_{k}}{\partial \lambda_{5}} \\
& =2 \lambda_{5}+2 \sum_{k=2}^{n}\left(\widehat{x}_{k}-x_{k}\right) d_{k}=0 .
\end{aligned}
$$

By solving (18), the following can be obtained:

$$
\hat{\lambda}_{5}=\frac{\sum_{k=2}^{n}\left(x_{k}-c_{k}-d_{k} x_{1}\right) d_{k}}{1+\sum_{k=2}^{n} d_{k}^{2}} .
$$

As such, the estimations of $\lambda_{1}, \lambda_{2}, \lambda_{3}, \lambda_{4}$, and $\lambda_{5}$ are all obtained. Let $k=1,2,3$ in (6), the simulation and forecasting results of the annual electricity consumption time series will be obtained.

\section{Model Test}

4.1. Data Selection and Preliminary Analysis. China is the largest electricity consumer among the developing countries. At present, the share of global electricity consumption in China is as high as $23.18 \%$ [2]. More importantly, electricity consumption in China is still in the phase of rapid growth. The precise forecasting of electricity consumption in China is important for the production planning of its power system. In this study, the electricity consumption data in China are used to test the performance of AHM.

Figure 1 shows the electricity consumption curve from the time of the founding of the People's Republic of China.

As shown in Figure 1, the long-term electricity consumption trend in China is an approximately exponential growth curve, except for some minor irregular waves. The growth speed of the curve is unstable, with linear or accelerated growth rate trends in certain periods. For example, from 1991 to 1997 , the annual increase amounts are 56.18, 76.70, 82.24, 91.45, 79.07, 72.41, and 54.85 TWh. These relatively stable increase amounts imply that the electricity consumption curve during this period nears a linear trend. However, the electricity consumption curve suddenly becomes very steep in the next several years. From 1998 to 2003, the annual growth rates are $2.07 \%, 6.52 \%, 10.98 \%, 8.43 \%, 10.06 \%$, and $16.53 \%$. That is, the electricity consumption curve roughly presents a steep trend with accelerated growth rates in this period. These above characteristics preliminary prove the necessity of the equation structure of AHM.

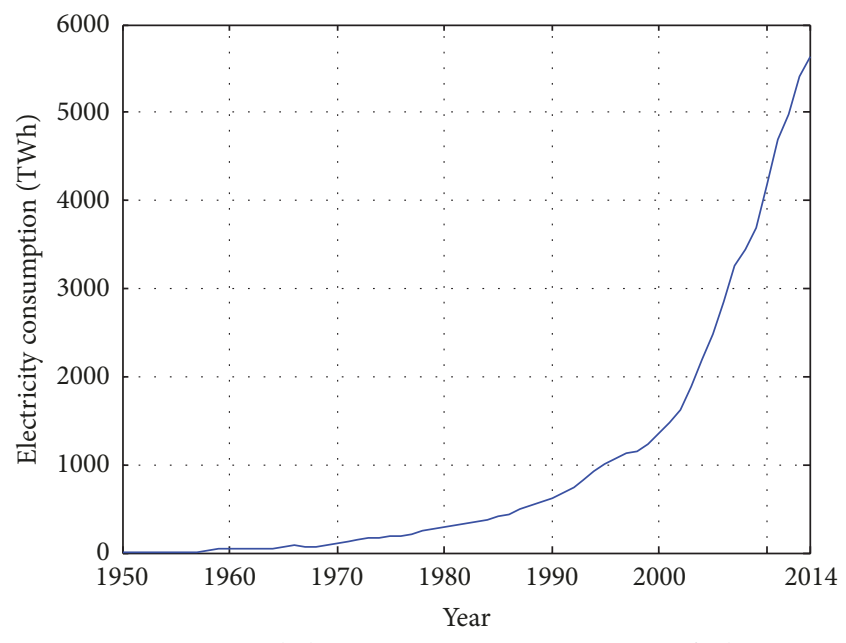

FIgURE 1: Annual electricity consumption curve of China.

4.2. Forecasting Results. To evaluate forecasting performance, ANN, GM(1,1), and the proposed AHM were all used to onestep-ahead forecast China's annual electricity consumption from 1991 to 2014, and then their results were compared.

Among the ANNs, the back-propagation (BP) and radial basis function (RBF) are the most represented models. While the latter is usually better than the former in terms of learning efficiency and stability [10], the RBF ANN was selected for this study. Before forecasting, 20 consecutive previous values of the forecasting point were selected to train the network. Among the 20 selected values, the 11th to 20 th values were selected as the output values, respectively, while ten previous values of each output value were selected as its input vector. After training, the forecasting result was obtained by inputting the 11th to 20 th values as the input vector into the trained network.

In forecasting using $\operatorname{GM}(1,1)$ and $\mathrm{AHM}, 10$ consecutive previous values of each forecasting point were selected to build the forecasting equation. For example, in forecasting electricity consumption in China in 1991, the electricity consumption values from 1981 to 1990 were selected to estimate the equation parameters of $\operatorname{GM}(1,1)\left[\beta_{1}\right.$ and $\beta_{2}$ in (1)] and $\operatorname{AHM}\left[\lambda_{1}\right.$ to $\lambda_{5}$ in (6)]. Let $k=10$ in (1) and (6), the forecasting results of $\operatorname{GM}(1,1)$ and AHM on the electricity consumption in China in 1991 were obtained.

The real data (RD, unit: TWh) of the annual electricity consumption in China from 1991 to 2014, the forecasting results of the three models (unit: TWh), and the relative error (RE, unit: \%) of each forecasting result are all listed in Table 1.

Table 1 shows that, compared with $\operatorname{GM}(1,1)$ and $\mathrm{AHM}$, the large errors of ANN appear more irregular. This finding implies that ANN is more easily affected by the stochastic changes of the annual electricity consumption curve than the other two models. $\mathrm{GM}(1,1)$ and AHM usually present large errors at the same time. Furthermore, compared with $\operatorname{GM}(1,1)$, AHM can always more quickly reduce the forecasting errors. This finding proves the adaptive ability of AHM. The curves of the $\mathrm{RD}$ and the forecasting results of the three models are drawn in Figure 2 to clearly demonstrate the forecasting performance. 
TABLE 1: Real electricity consumption data from 1991 to 2014, the forecasting results of ANN, GM(1, 1), and AHM, and the relative errors.

\begin{tabular}{|c|c|c|c|c|c|c|c|}
\hline & $\mathrm{RD}$ & ANN & $\mathrm{RE}$ & $\operatorname{GM}(1,1)$ & $\mathrm{RE}$ & AHM & $\mathrm{RE}$ \\
\hline 1991 & 677.49 & 664.85 & -1.87 & 685.29 & 1.15 & 678.26 & 0.11 \\
\hline 1992 & 754.19 & 711.51 & -5.66 & 740.8 & -1.78 & 736.05 & -2.41 \\
\hline 1993 & 836.43 & 798.96 & -4.48 & 812.09 & -2.91 & 814.48 & -2.62 \\
\hline 1994 & 927.88 & 904.8 & -2.49 & 896.53 & -3.38 & 899.4 & -3.07 \\
\hline 1995 & 1006.95 & 1022.21 & 1.52 & 995.72 & -1.12 & 996.31 & -1.06 \\
\hline 1996 & 1079.36 & 1109.58 & 2.8 & 1096.34 & 1.57 & 1089.81 & 0.97 \\
\hline 1997 & 1134.2 & 1170.05 & 3.16 & 1192.98 & 5.18 & 1179.6 & 4 \\
\hline 1998 & 1157.7 & 1192.83 & 3.03 & 1273.03 & 9.96 & 1247.48 & 7.76 \\
\hline 1999 & 1233.14 & 1175.92 & -4.64 & 1317.55 & 6.85 & 1262.31 & 2.37 \\
\hline 2000 & 1368.48 & 1212.55 & -11.39 & 1364.19 & -0.31 & 1329.46 & -2.85 \\
\hline 2001 & 1483.86 & 1350.35 & -9 & 1448.81 & -2.36 & 1448.71 & -2.37 \\
\hline 2002 & 1633.15 & 1546.41 & -5.31 & 1554.46 & -4.82 & 1557.41 & -4.64 \\
\hline 2003 & 1903.16 & 1768.33 & -7.08 & 1697.36 & -10.81 & 1696.14 & -10.88 \\
\hline 2004 & 2197.14 & 2178.16 & -0.86 & 1944.53 & -11.5 & 1955.75 & -10.99 \\
\hline 2005 & 2494.04 & 2640.28 & 5.86 & 2284.09 & -8.42 & 2317.58 & -7.08 \\
\hline 2006 & 2858.8 & 2984.72 & 4.4 & 2684.31 & -6.1 & 2718.92 & -4.89 \\
\hline 2007 & 3271.18 & 3324.53 & 1.63 & 3151.88 & -3.65 & 3176.78 & -2.89 \\
\hline 2008 & 3454.14 & 3670.65 & 6.27 & 3665.48 & 6.12 & 3658.83 & 5.93 \\
\hline 2009 & 3703.22 & 3666.77 & -0.98 & 4037.55 & 9.03 & 3906.75 & 5.5 \\
\hline 2010 & 4193.45 & 3731.66 & -11.01 & 4347.19 & 3.67 & 4170.84 & -0.54 \\
\hline 2011 & 4700.09 & 4154.32 & -11.61 & 4749.84 & 1.06 & 4649.36 & -1.08 \\
\hline 2012 & 4976.26 & 4794.79 & -3.65 & 5217.07 & 4.84 & 5170.92 & 3.91 \\
\hline 2013 & 5420.34 & 5180.37 & -4.43 & 5597.49 & 3.27 & 5471.63 & 0.95 \\
\hline 2014 & 5638.37 & 5623.90 & -0.26 & 6020.41 & 6.78 & 5923.45 & 5.06 \\
\hline
\end{tabular}

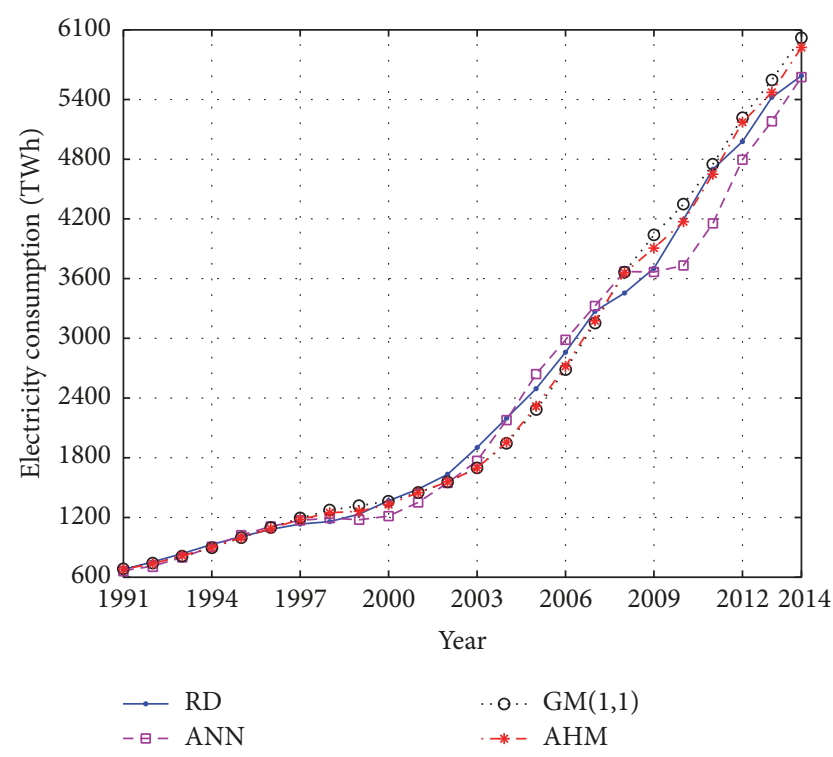

FIGURE 2: Curves of the RD and the forecasting results of the three models.

As shown in Figure 2, the rate of increase of the electricity consumption curve decreased unexpectedly in 1998 because of the Southeast Asian financial crisis and returned to its main trend after 2000. This can be identified as a stochastic change.
Figure 2 shows that this stochastic change significantly affected the forecasting precision of ANN and resulted in continuous large errors. By contrast, $\mathrm{GM}(1,1)$ and AHM were less affected by this unexpected change, especially AHM, which showed an immediate adjustment of the forecasting result and smaller errors were obtained since 1999.

In 2008, the rate of increase of the electricity consumption decreased again. Unlike in 1998, the RD curve in Figure 2 shows that the change caused a downshift to the main trend. The forecasting precision of ANN was again significantly affected. The forecasting results of AHM, by contrast, exhibited an immediate response, with the values becoming close to the $\mathrm{RD}$ since 2010. Compared with AHM, the response of $\operatorname{GM}(1,1)$ is evidently slower, and this makes it have larger forecasting errors.

The performance of AHM can be explained by its specialized forecasting equation. The basic items comprising the forecasting equation of AHM are all constant trend equations, thus allowing AHM to overcome the stochastic effects to the electricity consumption curve. Moreover, at the change in the main electricity consumption trend, AHM can quickly adapt through the adjustment of the parameters of the different items.

4.3. Error Analysis. To comprehensively evaluate the performance of the three forecasting models, the mean absolute percentage error (MAPE), median absolute percentage error 
TABLE 2: Results of the error analysis based on MAPE, MaxAPE, and MdAPE.

\begin{tabular}{lccc}
\hline & ANN & GM $(1,1)$ & AHM \\
\hline MAPE & 4.725 & 4.859 & 3.912 \\
MaxAPE & 11.612 & 11.497 & 10.987 \\
MdAPE & 4.416 & 4.242 & 2.978 \\
\hline
\end{tabular}

(MdAPE), and maximal absolute percentage error (MaxAPE) were used as the indicators to measure the forecasting precision. These indicators are defined as follows:

$$
\begin{aligned}
& \text { MAPE }=\operatorname{mean}_{k}\left(\left|\frac{\hat{x}_{k}-x_{k}}{x_{k}}\right|\right) \cdot 100, \\
& k=1,2, \ldots, n, \\
& \operatorname{MaxAPE}=\max _{k}\left(\left|\frac{\widehat{x}_{k}-x_{k}}{x_{k}}\right|\right) \cdot 100, k=1,2, \ldots, n, \\
& \operatorname{MdAPE}=\operatorname{median}_{k}\left(\left|\frac{\hat{x}_{k}-x_{k}}{x_{k}}\right|\right) \cdot 100, \\
& k=1,2, \ldots, n .
\end{aligned}
$$

These indicators have similar functions in evaluating the performance of each forecasting model, but they still have fine distinctions. MAPE is the most widely used indicator, which can reflect the general closeness of the forecasting results to the RD. In addition to having functions similar to those of MAPE in measuring the general closeness of the forecasting results to the $\mathrm{RD}, \mathrm{MdAPE}$ can overcome the influence of several outliers. MaxAPE records the worst forecasting result and reflects the maximal forecasting risk.

Using the data in Table 1 and (20) the results for the error analysis based on the three indicators were obtained and are shown in Table 2.

As shown in Table 2, AHM is better than ANN and GM(1, 1) based on each indicator. This finding proves that compared with $\mathrm{ANN}$ and $\operatorname{GM}(1,1)$, the forecasting results of AHM are not only generally closer to the real data, but also have less forecasting risk.

\section{Conclusions}

This study first presented a small-sample AHM for annual electricity consumption forecasting. The proposed iterative equation, which contained linear, exponential, and steep items, was used for trend extrapolation of the possible changes of the electricity consumption curve. The PLS algorithm was employed to estimate the parameters of the iterative equation to improve forecasting stability using small samples. Furthermore, the parameter of the iteration starting point was obtained by minimizing the RSS. To evaluate forecasting performance, $\mathrm{ANN}, \mathrm{GM}(1,1)$, and the proposed $\mathrm{AHM}$ are used to forecast China's electricity consumption from 1991 to 2014, and then their results are compared. With the forecasting equation of the proposed AHM composed of three constant trend terms, the forecasting results of AHM are less affected by stochastic change than those of ANN. Moreover, at the change in the main electricity consumption trend, the forecasting results of the proposed AHM showed a more immediate adjustment than $\operatorname{GM}(1,1)$ because of the specialized equation of AHM that can simulate three trends at the same time. To comprehensively evaluate the forecasting precision, MAPE, MdAPE, and MaxAPE of each model were calculated. All indicators showed that AHM is better than ANN and $\mathrm{GM}(1,1)$.

\section{Notations}

$x_{k}$ : The $k$ th values of the annual electricity consumption time series

$\widehat{x}_{k}$ : The fitting/forecasting result to $x_{k}$

$F_{0}$ : The standardized vector of observations of independent variable

$E_{0}$ : The standardized matrix of observations of dependent variables

$t_{1}$ : The extracted component which is the weighted combination of the columns of $E_{0}$

$F_{1}$ : The residual vector obtained by using $t_{1}$ to explain $F_{0}$

$E_{1}$ : The residual matrix obtained by using $t_{1}$ to explain $E_{0}$

$Q_{h}^{2}$ : The indicator to evaluate the importance of a extracted component.

\section{Conflicts of Interest}

The authors declare that there are no conflicts of interest regarding the publication of this paper.

\section{Acknowledgments}

The authors are grateful for the financial support provided by the National Natural Science Foundation of China (NSFC) (71471061 and 71201057).

\section{References}

[1] X. Xu, D. Niu, M. Meng, and H. Shi, "Yearly electricity consumption forecasting using a nonhomogeneous exponential model optimized by PSO algorithm," Applied Mathematics \& Information Sciences, vol. 8, no. 3, pp. 1063-1069, 2014.

[2] BP, "Statistical Review of World Energy," http://www.bp.com/ statisticalreview.

[3] M. Meng and D. Niu, "Annual electricity consumption analysis and forecasting of China based on few observations methods," Energy Conversion and Management, vol. 52, no. 2, pp. 953-957, 2011.

[4] V. Bianco, O. Manca, and S. Nardini, "Linear regression models to forecast electricity consumption in Italy," Energy Sources, Part B: Economics, Planning and Policy, vol. 8, no. 1, pp. 86-93, 2013.

[5] J. Wang, J. Zhang, and J. Nie, "An improved artificial colony algorithm model for forecasting chinese electricity consumption and analyzing effect mechanism," Mathematical Problems in Engineering, vol. 2016, Article ID 8496971, 14 pages, 2016.

[6] J. Wang, L. Li, D. Niu, and Z. Tan, "An annual load forecasting model based on support vector regression with differential evolution algorithm," Applied Energy, vol. 94, pp. 65-70, 2012. 
[7] H.-Z. Li, S. Guo, C.-J. Li, and J.-Q. Sun, "A hybrid annual power load forecasting model based on generalized regression neural network with fruit fly optimization algorithm," KnowledgeBased Systems, vol. 37, pp. 378-387, 2013.

[8] J. Olauson and M. Bergkvist, "Modelling the Swedish wind power production using MERRA reanalysis data," Renewable Energy, vol. 76, pp. 717-725, 2015.

[9] D. J. Pedregal and P. C. Young, "Development of improved adaptive approaches to electricity demand forecasting," Journal of the Operational Research Society, vol. 59, no. 8, pp. 1066-1076, 2008.

[10] Z. Zhang and W. Gong, "Short-term load forecasting model based on quantum Elman neural networks," Mathematical Problems in Engineering, vol. 2016, Article ID 7910971, 8 pages, 2016.

[11] Y. Ren, P. N. Suganthan, N. Srikanth, and G. Amaratunga, "Random vector functional link network for short-term electricity load demand forecasting," Information Sciences, vol. 367-368, pp. 1078-1093, 2016.

[12] Y. Ohtsuka and K. Kakamu, "Space-time model versus VAR model: forecasting electricity demand in Japan," Journal of Forecasting, vol. 32, no. 1, pp. 75-85, 2013.

[13] H. Cui and X. Peng, "Short-term city electric load forecasting with considering temperature effects: an improved ARIMAX model," Mathematical Problems in Engineering, vol. 2015, Article ID 589374, 10 pages, 2015.

[14] Z.-X. Wang, "A genetic algorithm-based grey method for forecasting food demand after snow disasters: an empirical study," Natural Hazards, vol. 68, no. 2, pp. 675-686, 2013.

[15] M. Evans, "An alternative approach to estimating the parameters of a generalised Grey Verhulst model: an application to steel intensity of use in the UK," Expert Systems with Applications, vol. 41, no. 4, pp. 1236-1244, 2014.

[16] B. Zeng and C. Li, "Forecasting the natural gas demand in China using a self-adapting intelligent grey model," Energy, vol. 112, pp. 810-825, 2016.

[17] A. I. Köne and T. Büke, "Forecasting of $\mathrm{CO}_{2}$ emissions from fuel combustion using trend analysis," Renewable and Sustainable Energy Reviews, vol. 14, no. 9, pp. 2906-2915, 2010.

[18] R. H. Hoyle, Statistical Strategies for Small Sample Research, SAGE, Los Angeles, Calif, USA, 1999.

[19] H. Zhang, J. Guo, J. Zhu, and J. Yu, Multivariate Data Analysis Methods and Applications with Few Observations, Northwestern Polytechnical University Press, Xi'an, China, 2002 (Chinese).

[20] Y. Zhang, Z. Wang, and G. Zhou, "Determinants and implications of employee electricity saving habit: an empirical study in China," Applied Energy, vol. 112, no. 4, pp. 1529-1535, 2013.

[21] H. Wang, Z. Wu, and J. Meng, Partial Least Squares RegressionLinear and Nonlinear Methods, National Defense Industry Press, Beijing, China, 2006 (Chinese). 


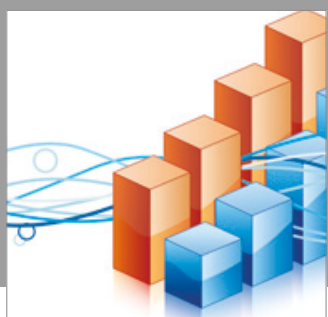

Advances in

Operations Research

vatersals

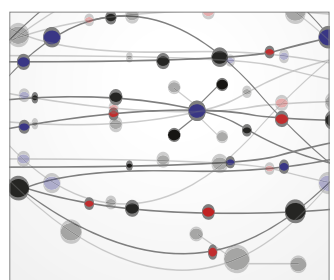

\section{The Scientific} World Journal
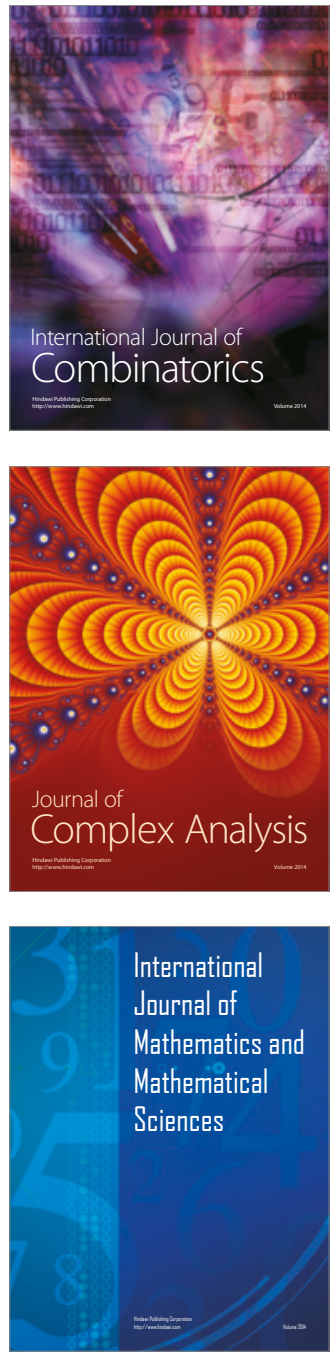
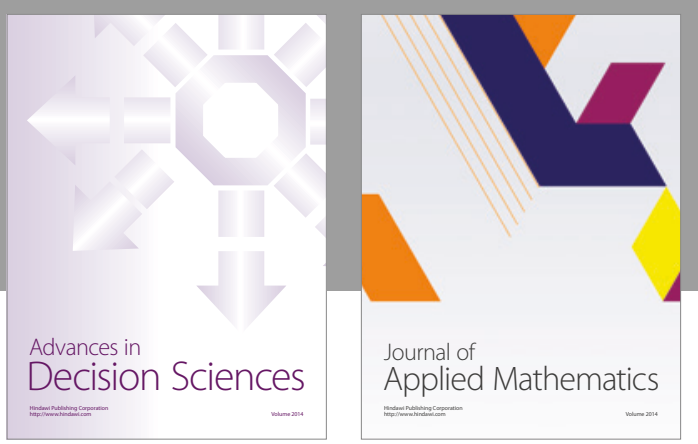

Algebra

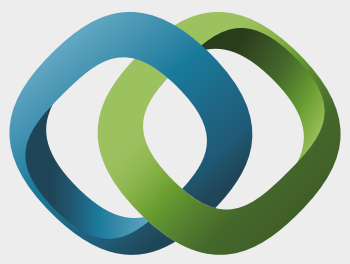

\section{Hindawi}

Submit your manuscripts at

https://www.hindawi.com
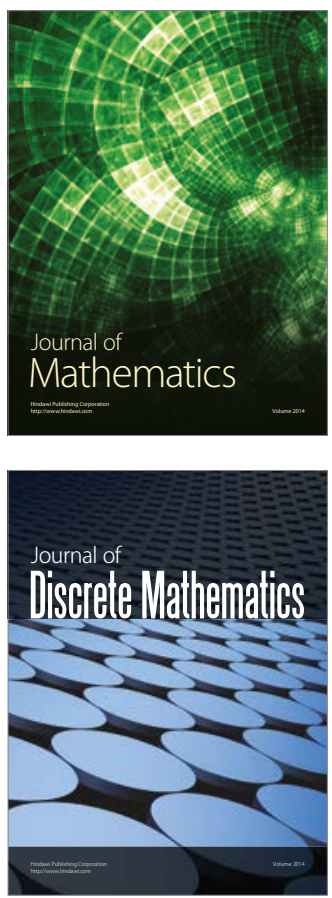

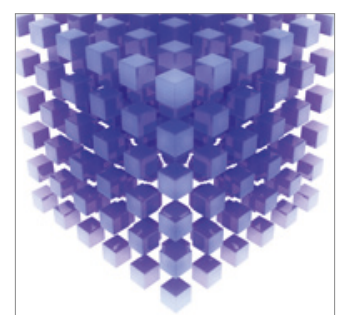

Mathematical Problems in Engineering
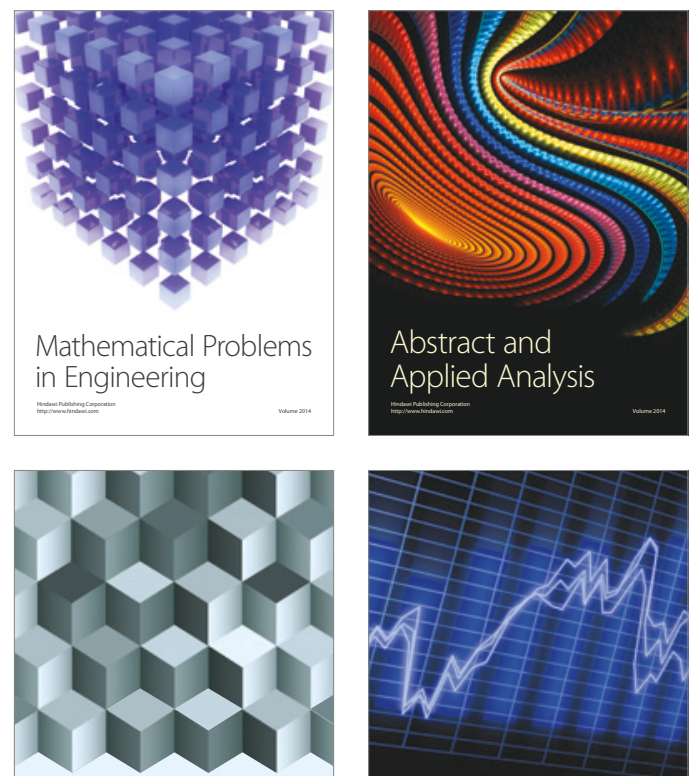

Journal of

Function Spaces

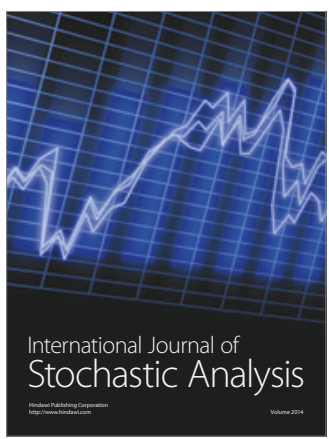

Probability and Statistics
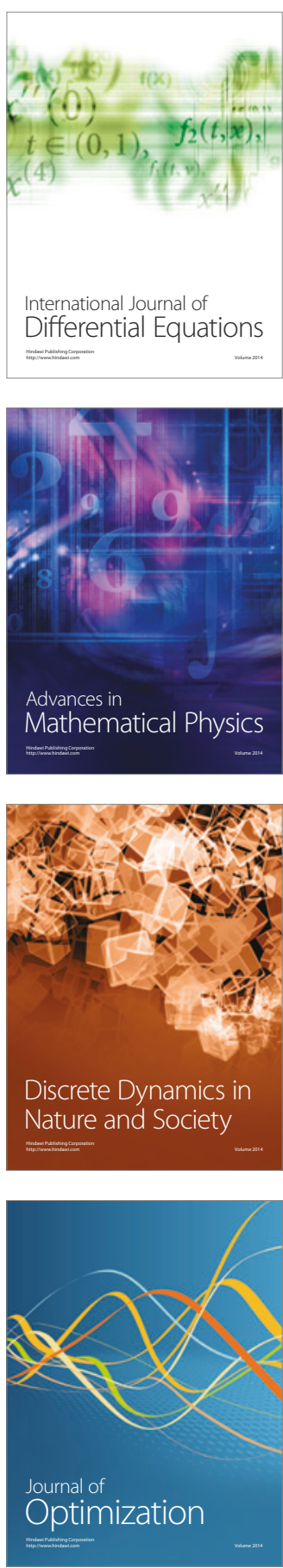